\title{
Acute embolisation of MitraClip rescued by snaring
}

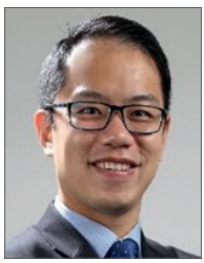

Ningyan Wong*, MBBS, MRCP, M.Med; See Hooi Ewe, MBBS, MRCP, PhD;

Wei Chieh Jack Tan, MBBS, MRCP, M Med, FAMS, ACSM, FAsCC, FAPSIC, FESC, FACC, MBA; Khung Keong Yeo, MBBS, FAMS, FACC, FSCAI, ABIM

Department of Cardiology, National Heart Centre Singapore, Singapore

This paper also includes supplementary data published online at: www.asiaintervention.org

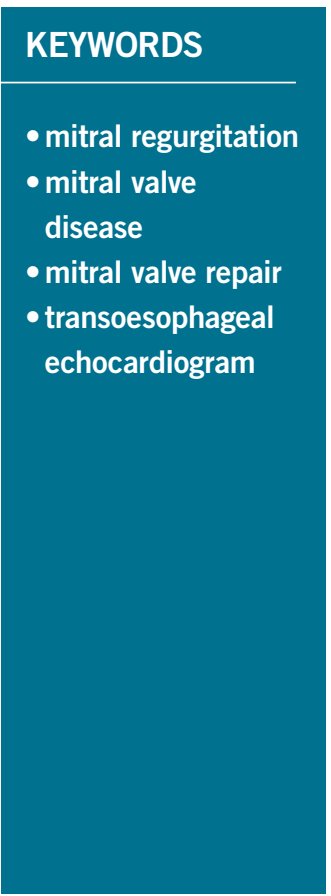

\section{Abstract}

Percutaneous edge-to-edge repair of the mitral valve with the MitraClip system is a safe and effective treatment option for patients with severe degenerative mitral regurgitation who are at prohibitive surgical risk. However, although uncommon, complications do happen and one of which is device embolisation. We describe a case of complete dislodgement of MitraClip that occurred periprocedurally and how this can be rescued percutaneously using a snare.

*Corresponding author: National Heart Centre Singapore, 5 Hospital Drive, Singapore 169609.

E-mail: wong.ningyan@singhealth.com.sg 


\section{Abbreviations}

MR

TOE

LA

mitral regurgitation

transoesophageal echocardiogram

left atrium

\section{Introduction}

Transcatheter mitral valve repair with the MitraClip ${ }^{\mathrm{TM}}$ (Abbott Vascular, Santa Clara, CA, USA) system is a treatment option for patients with severe degenerative mitral regurgitation (MR) at prohibitive surgical risk. Earlier trials had demonstrated its excellent safety profile, but complications can happen. We describe a case of periprocedural complete dislodgement of the MitraClip and how it was successfully rescued percutaneously with a snare, followed by its retrieval through a surgical cutdown of the common femoral vein access.

\section{Methods}

An 83-year-old Chinese gentleman with stage three chronic kidney disease, idiopathic thrombocytopaenic purpura and chronic anaemia presented with recurrent heart failure from severe MR. Transoesophageal echocardiogram (TOE) showed Barlow's disease - bileaflet mitral valve prolapse involving A2/P2, A3/P3, with predominant prolapse of the $\mathrm{A} 3 / \mathrm{P} 3$ segments and a small ruptured chord at the tip of the posteromedial segment (Figure 1, Moving images 1-Moving image 3), resulting in severe eccentric MR. After a Heart Team discussion, in view of increased age and multiple comorbidities, he was deemed to be at high surgical risk (EuroSCORE II and STS scores for mortality were $7.3 \%$ and $22.1 \%$, respectively). He was hence offered percutaneous repair of the mitral valve with the MitraClip system.

The first clip was deployed at the A3/P3 segments. Although this reduced the MR severity from $4+$ to $3+$, it was still substantial lateral to the clip (Moving image 4). A decision was made to deploy a second clip. Prior to clip release, the anterior and posterior leaflet grasps were imaged with adequate tissue seen within the clip (Moving image 5). After releasing the lock line, the second clip detached immediately from the posterior leaflet but remained attached to the anterior leaflet (Moving image 6, Moving image 7). At this time, the team decided to deploy the clip and rescue the situation with a third clip lateral to the second clip to stabilise it. However, after the deployment pin was released, the entire clip detached from the mitral valve and was held in the left atrium (LA) by the gripper line (Figure 2, Moving image 8-Moving image 10). A decision was made to snare the clip with bail-out surgery as an option. First, the guide catheter was withdrawn into the right atrium to reduce the risk of air embolism. A 0.035 " wire was placed into the LA via the same transseptal hole. Initially the $9-15 \mathrm{~mm}$ EN Snare ${ }^{\circledR}$ (Merit Medical Systems, Inc. South Jordan, UT, USA) was used but it failed to grasp the clip (Moving image 11). It was changed to a $25 \mathrm{~mm}$ Amplatz GooseNeck $^{\circledR}$ snare (ev3, Plymouth, MN, USA) via an 8 Fr guide and we were successful in snaring it (Moving image 12). The clip

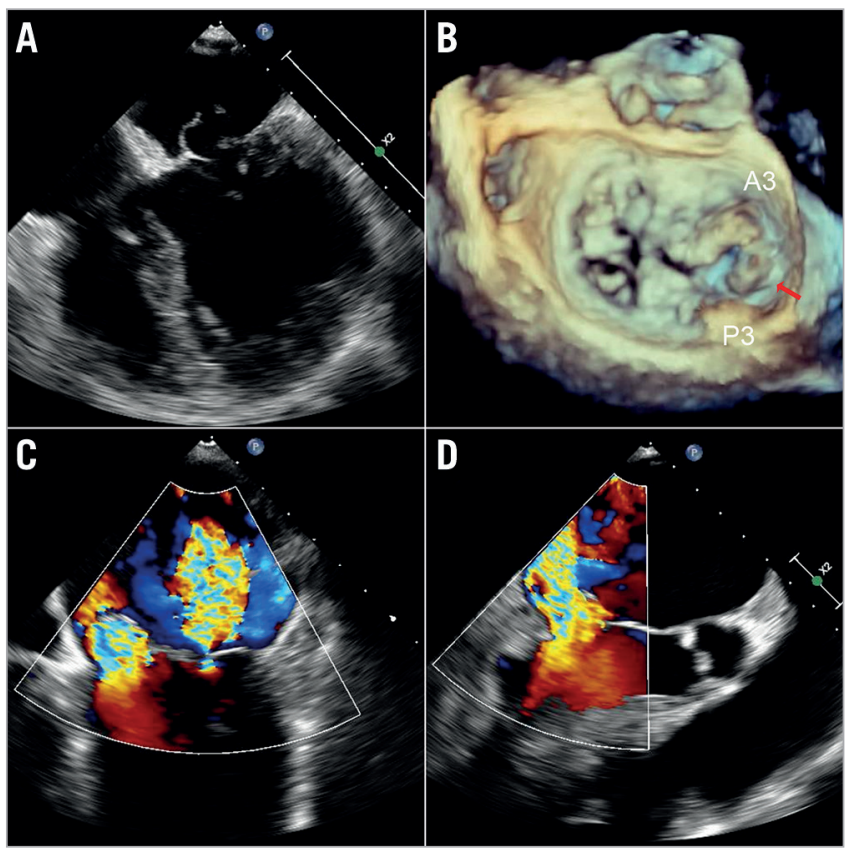

Figure 1. Bileaflet mitral valve prolapse with severe mitral regurgitation (MR). A, B) Transoesophageal echocardiogram (TOE) images of prolapsed segments A2/P2, A3/P3 with predominant prolapse of A3/P3 and a small ruptured chord at the tip of the posteromedial segment (red arrow). Severe MR in the apical 4-chamber (C) and the left ventricular outflow tract (LVOT) view (D).
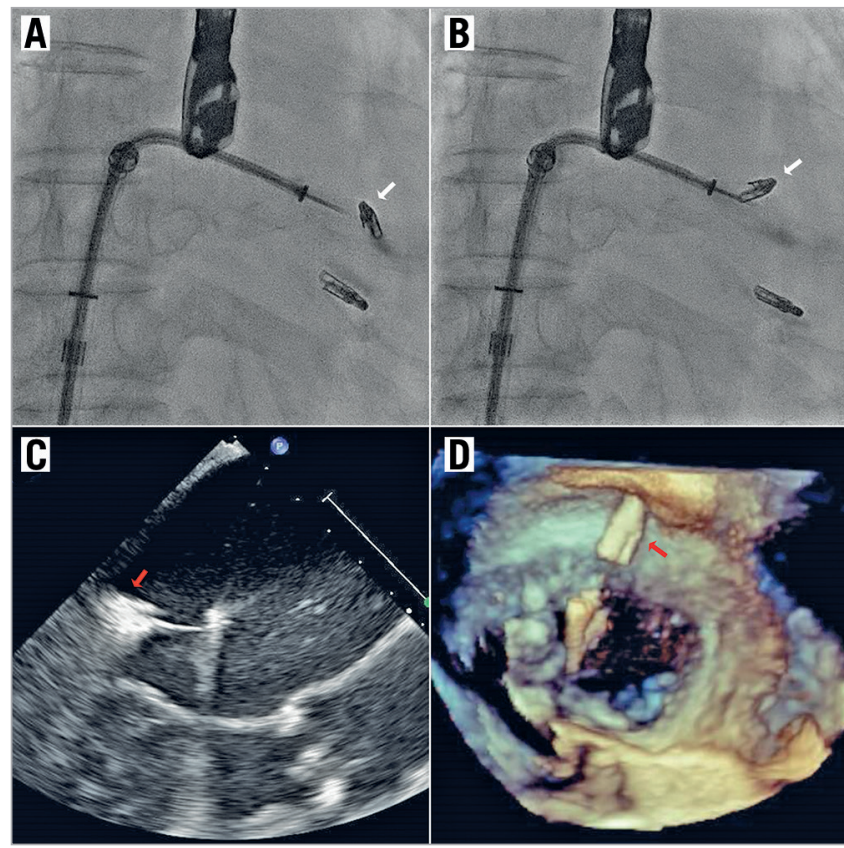

Figure 2. MitraClip embolisation. A, B) Fluoroscopic images showing complete detachment of the 2nd MitraClip (white arrows) after deployment but held within the left atrium by the gripper line. TOE images of the dislodged MitraClip (red arrows) in the LVOT view $(C)$ and the en face $3 D$ view where it is held above the mitral valve by the gripper line $(D)$. 
was pulled back across the inter-atrial septum and down into the femoral vein (Figure 3). A surgical cutdown to the common femoral vein was performed to retrieve the clip (Figure 4).
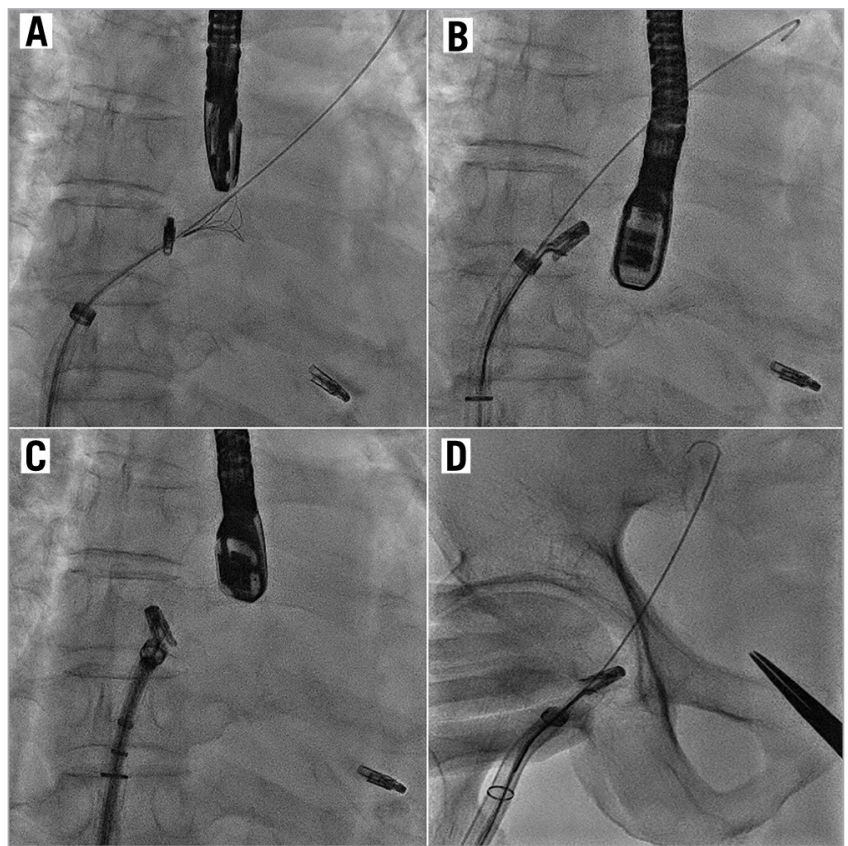

Figure 3. Snaring of embolised MitraClip. Fluoroscopic images showing (A) an unsuccessful attempt to snare the MitraClip with the EN Snare. B) The GooseNeck snare was successful in snaring the clip. The Clip was pulled across the interatrial septum (C) and to the common femoral vein (D).

The mitral valve was reassessed and there were no signs of new torn chords or flail segments. There was residual moderate to severe MR (Figure 5, Moving image 13). At this time, given the circumstances and that there was no worsening of MR, a decision was made to stop.

\section{Results}

The patient was discharged well four days after the procedure. He clinically improved to New York Heart Association Class I with

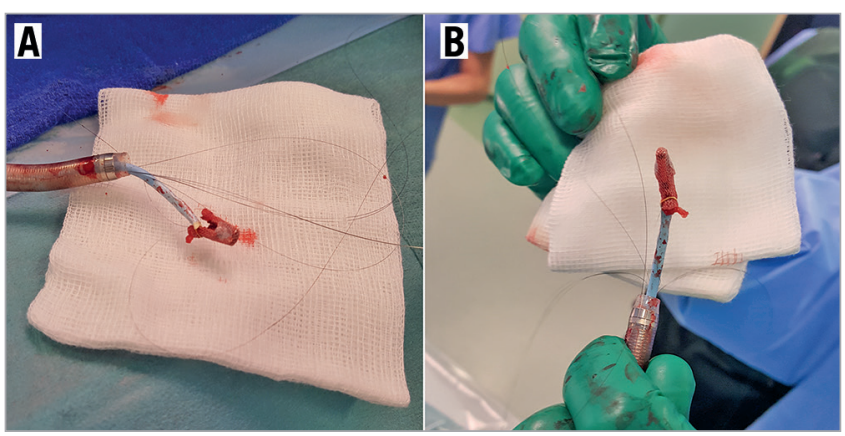

Figure 4. Embolised MitraClip removed. A, B) Photographic images of the MitraClip removed from the patient, with the GooseNeck snare still attached to one of the clip arms.

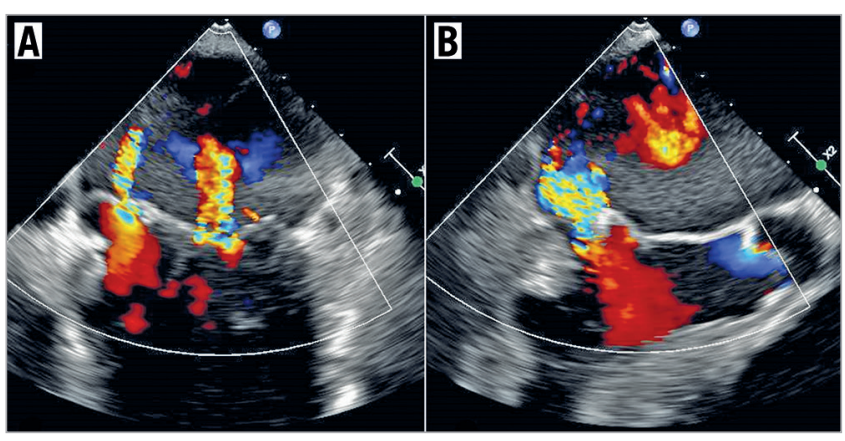

Figure 5. Residual MR. TOE images of residual moderate to severe $M R$ in the intercommissural view (A) and the LVOT view (B), with just a single MitraClip deployed. There was no evidence of torn leaflets seen.

no further heart failure exacerbation. His follow-up echocardiogram at one month, six months and one year showed stable residual moderate to severe MR.

\section{Discussion}

Clip detachment is a potential complication of using the MitraClip system. Single leaflet detachment occurs more commonly but complete detachment and embolisation is exceedingly rare with only a few cases reported to date ${ }^{1-5}$. MitraClip operators must be familiar with handling this complication.

The mechanism of early periprocedural clip detachment is usually due to inadequate leaflet capture or insertion. In our case, despite what appeared to be adequate grasping of both leaflets, there was early detachment initially of the posterior leaflet, and subsequently the anterior leaflet. Other possibilities include tearing of the leaflet, grasping of chordae instead of the leaflet and slippage of the leaflet from the clip and grippers. In this case, leaflet tearing was unlikely as there was no worsening of MR or visible torn leaflets. Our clinical suspicion is that while we had grasped the leaflets, there was probably insufficient capture resulting in subsequent slippage and embolisation.

The MitraClip gripper line acts as a final fail-safe mechanism in the event of a completely detached clip. Because the clip was still attached to the gripper line, it prevented complete embolisation of the device and could thus be rescued with a snare. We used a 0.035 " wire to ensure access to the LA and via the wire we were able to deliver the snare. The EN Snare shape was not suitable in this case as it was difficult to get the 'flower' shaped snare to grasp the embolised clip. On the other hand, it was easier to get the GooseNeck snare distal to the clip and by pulling back against the catheter, we were able to close the snare and capture the clip. Another possibility was to pull both ends of the gripper line in an attempt to retrieve the clip. However, we felt that this method would not give us adequate control to pull the clip across the interatrial septum and into the guiding catheter. Secondly, there was a risk of losing the clip completely if the nitinol gripper line stretched and broke. 


\section{Conclusion}

In conclusion, adequate leaflet insertion into each clip arm is essential. In the event that complete clip dislodgement occurs, it is crucial not to lose the gripper line as it can function as a final safety mechanism to prevent systemic embolisation. Snaring allows for a percutaneous avenue to rescue the dislodged clip.

\section{Impact on daily practice}

Clip embolisation is a potential complication of using the MitraClip system and this can happen periprocedurally. Snaring is a percutaneous option to rescue the dislodged clip and MitraClip operators must be familiar with handling this complication.

\section{Conflict of interest statement}

Yeo Khung Keong is a speaker, consultant and proctor for Abbott Vascular (MitraClip) and reports receiving honoraria from Abbott Vascular. The other authors have no conflicts of interest to declare.

\section{References}

1. Chitsaz S, Jumean M, Dayah T, Rajagopal K, Kar B. Late MitraClip Embolization: A New Cause of ST-Segment-Elevation Myocardial Infarction. Circ Cardiovasc Interv. 2016;9 :e004271.

2. Bilge M, Alsancak Y, Ali S, Duran M, Biçer H. An extremely rare but possible complication of MitraClip: embolization of clip during follow-up. Anatol J Cardiol. 2016;16:636-8.

3. Seeburger J, Raschpichler M, Lurz P, Noack T, Ender J, Misfeld M. Late device embolization after transcatheter mitral valve edge-to-edge repair. Eur Heart J. 2017;38:1260.

4. Alozie A, Westphal B, Kische S, Kaminski A, Paranskaya L, Bozdag-Turan I, Ortak J, Schubert J, Steinhoff G, Ince H. Surgical revision after percutaneous mitral valve repair by edge-to-edge device: when the strategy fails in the highest risk surgical population. Eur J Cardiothorac Sur. 2014;46:55-60

5. Pasala TKR, Safi LM, Jelnin V, Ruiz CE. Catching a "MitraFly". JACC Cardiovasc Interv. 2018 Jun 25;11:1201-3.

\section{Supplementary data}

Moving image 1. Preoperative transoesophageal echocardiogram (TOE) showing marked prolapse of anterior mitral valve leaflet.

Moving image 2. Preoperative TOE with colour Doppler in the apical 4-chamber view showing severe mitral regurgitation (MR), predominantly from A3 with a smaller jet from A2.

Moving image 3. Preoperative TOE in $3 \mathrm{D}$ view showing prolapsed segments $\mathrm{A} 2 / \mathrm{P} 2, \mathrm{~A} 3 / \mathrm{P} 3$ with predominant prolapse of $\mathrm{A} 3 / \mathrm{P} 3$ and a small ruptured chord at the tip of the posteromedial segment. Moving image 4. TOE with colour Doppler showing at least moderate MR lateral to the first MitraClip.

Moving image 5. TOE showing good grasp of the mitral valve leaflets with significant reduction in MR.

Moving image 6. Fluoroscopic imaging showing deployment of the second MitraClip with immediate partial detachment from the posterior leaflet.

Moving image 7. TOE showing detachment of the second MitraClip from the posterior leaflet, but the clip remains attached to the anterior leaflet.

Moving image 8. Fluoroscopic imaging showing complete detachment of the second MitraClip.

Moving image 9. TOE showing the second MitraClip completely detached from the leaflets but remaining attached to the gripper line.

Moving image 10. TOE in 3D view showing complete detachment of the second MitraClip.

Moving image 11. Unsuccessful snaring of the dislodged MitraClip with an EN Snare.

Moving image 12. Successful snaring of the dislodged MitraClip with a Gooseneck snare.

Moving image 13. TOE with colour Doppler in the intercommissural view showing residual moderate to severe MR with no tearing of the mitral valve leaflet seen.

The supplementary data are published online at: www.asiaintervention.org 